\title{
2016 Chevalley Prize in Lie Theory
}

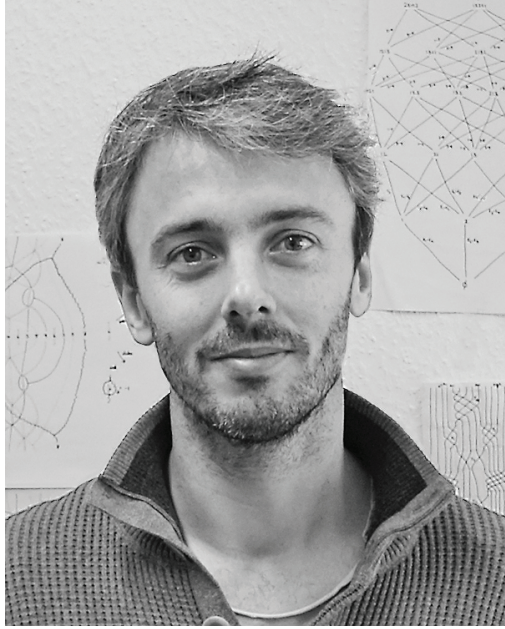

Geordie Williamson
GEORDIE WILLIAMSON was awarded the 2016 Chevalley Prize in Lie Theory at the 122nd Annual Meeting of the AMS in Seattle, Washington, in January 2016.

\section{Citation}

The 2016 inaugural Chevalley Prize in Lie Theory is awarded to Geordie Williamson for his work on the representation theory of Lie algebras and algebraic groups. His results include proofs and reproofs of some long-standing conjectures, as well as spectacular counterexamples to the expected bounds in others.

The characters of finite-dimensional simple modules for semisimple complex Lie algebras have been known for, by now, about ninety years, thanks to the work of Hermann Weyl. For certain natural infinite-dimensional generalizations, the simple modules with a highest weight, a character formula was conjectured by Kazhdan and Lusztig in 1979 and shortly after proved by Beilinson and Bernstein and (independently) by Brylinski and Kashiwara. These proofs relied on translating the problem in several steps to different areas of mathematics, further and further away from the original problem that is completely algebraic in nature. Williamson, in joint work with Ben Elias, has developed a purely algebraic Hodge theory for certain (Soergel) bimodules over polynomial rings. This has led to the first algebraic proof of the Kazhdan-Lusztig conjectures and a great simplification of the whole theory.

A most striking and new aspect of this new algebraic Hodge theory is that it applies to any

For permission to reprint this article, please contact: reprint-permission@ams.org.

DOI: http://dx.doi.org/10.1090/noti1352
Coxeter group, finite or infinite. An immediate consequence is a proof of various positivity conjectures concerning Kazhdan-Lusztig polynomials, which had been open since 1979 . Thanks to these new ideas, we can now treat general Coxeter groups and their Hecke algebras as if they had a geometric construction, even though the geometric objects (flag varieties) do not exist.

Williamson's next breakthrough concerned representations of reductive groups over fields of characteristic $p>0$. In 1980 Lusztig conjectured a character formula for the simple modules for these groups under a minor restriction on $p$. (For a group such as $\operatorname{GL}(n, F), p>2 n$ is assumed.)

This conjecture was proved in 1994 for all $p$ larger than a bound depending on the type of the group, first without an explicit bound (Andersen, Jantzen, Soergel) and later with a gigantic bound (Fiebig). Williamson has now stunned the experts by finding several infinite families of counterexamples to the expected bounds in Lusztig's conjecture, beginning with the special linear group SL(22, F). At the same time, he found counterexamples to James's 1990 conjecture on symmetric groups. More importantly, Williamson did not just provide counterexamples, he provided a new framework for thinking about these conjectures-a framework that revealed how inadequate the numerical evidence for these conjectures really had been. Williamson's work has reopened the field of modular representations to new ideas, in a sense taking it beyond a focus on the famous conjectures.

Williamson's work on these topics appears in "The Hodge theory of Soergel bimodules", Ann. of Math. (2) 180 (2014), 1089-1136 (with B. Elias); "Schubert calculus and torsion explosion", arXiv:1309.5055v2 (with an appendix by A. Kontorovich, P. McNamara, and G. Williamson); and "Modular intersection cohomology complexes on flag varieties", Math. Z. 272 (2012), no. 3-4, 697-727 (with an appendix by Tom Braden).

\section{Biographical Sketch}

Geordie Williamson was born in Bowral, Australia, in 1981. He received a bachelor of arts with honors and the University Medal from Sydney University in 2003. In 2008 he completed his PhD 
at the University of Freiburg, Germany, under the supervision of Wolfgang Soergel. He went on to spend three years at the University of Oxford as an EPSRC postdoctoral fellow and junior research fellow at St. Peter's College. Since 2011 he has been an advanced researcher at the Max Planck Institute for Mathematics in Bonn, and he is a principal research fellow at the University of Sydney. He works on problems in representation theory, algebra, and geometry and has given invited lectures throughout the world, including at MIT, MSRI, Orsay, RIMS, Sanya, and Yale. In 2016 he will deliver an invited lecture at the European Congress of Mathematics.

\section{Response from Geordie Williamson}

I am greatly honoured to receive the 2016 Chevalley Prize in Lie Theory. I would like to express my gratitude to George Lusztig, who both founded this prize and created so much of the field in which I work. Lie theory would be the poorer without his many observations, theorems, ideas, and conjectures. Chevalley's work is in good hands! Many thanks to my mentors and collaborators (in particular Wolfgang Soergel, Daniel Juteau, and Ben Elias) for all that they have taught me and the many hours we have spent together trying to understand a little better. I would like to thank the Max Planck Institute in Bonn and the University of Sydney for providing me with the space and means to concentrate on research. Finally, I thank my family and friends for their support and encouragement.

\section{About the Prize}

The Chevalley Prize in Lie Theory is awarded for notable work in Lie theory published during the preceding six years; a recipient should be no more than twenty-five years past the $\mathrm{PhD}$. The current prize amount is US $\$ 8,000$, awarded in even-numbered years, without restriction on society membership, citizenship, or venue of publication. The Chevalley Prize in Lie Theory was established in 2014 by George Lusztig to honor Claude Chevalley (1909-84). Chevalley was a founding member of the Bourbaki group. He made fundamental contributions to class field theory, algebraic geometry, and group theory. His three-volume treatise on Lie groups served as a standard reference for many decades. His classification of semisimple groups over an arbitrary algebraically closed field provides a link between Lie's theory of continuous groups and the theory of finite groups, to the enormous enrichment of both subjects.

The prize committee for the inaugural Chevalley Prize consisted of the following individuals.

- James G. Arthur

- Jens Carsten Jantzen (Chair)

- Michele Vergne

\section{a I[ЕГП}

Institute for Computational and Experimental Research in Mathematics

\section{CALL FOR PROPOSALS}

The Institute for Computational and Experimental Research in Mathematics (ICERM) at Brown University is seeking proposals for programs that support its mission to foster and broaden the relationship between mathematics and computation. We welcome pre and full proposals for semester programs, topical workshops, Summer@ICERM (undergraduate research), and Collaborate@ICERM (small team research).

SEMESTER PROGRAMS: ICERM hosts 2 semester programs per year. Each has 5-10 organizers and typically incorporates 3 week-long associated workshops. Semester program proposers should contact the ICERM director to discuss program ideas prior to starting the proposal process (director@icerm.brown.edu).

TOPICAL WORKSHOPS: ICERM hosts several topical workshops each year. These workshops typically last 5 days and focus on a timely and exciting theme of interest. Proposal requirements include a 2 page document that describes the scientific goals, lists the program organizers, and identifies the key participants.

SUMMER@ICERM: This is an 8 week undergraduate research program typically with 2 faculty leaders and several TAs in residence. Pre-proposal requirements include a 2-3 page document which identifies 1 faculty leader and potential partner(s), a short description of the focus of the program, and a sample list of 8-10 research projects suitable for undergraduates.

COLLABORATE@ICERM: This small group research program offers teams of 3-6 researchers the opportunity to spend 5 days at the institute. Proposal requirements include a 2-3 page description of the research project, an explanation for why ICERM is the ideal place to meet, and specifies members of the team.

DETAILS AND DEADLINES: For more details about the pre and full proposal process, requirements, and deadlines, please visit the ICERM "Proposal" website: icerm.brown.edu/get_involved/

About ICERM: The Institute for Computational and Experimental Research in Mathematics is a National Science Foundation Mathematics Institute at Brown U University in Providence, Rhode Island. Its mission is to broaden the relationship between mathematics and computation.

121 S. Main Street | Providence, Rl| 02903

401-863-5030 ||www.icerm.brown.edu 